\title{
Distributed Halide
}

\author{
Tyler Denniston \\ MIT \\ tyler@csail.mit.edu
}

\author{
Shoaib Kamil \\ Adobe \\ kamil@adobe.com
}

\author{
Saman Amarasinghe \\ MIT \\ saman@csail.mit.edu
}

\begin{abstract}
Many image processing tasks are naturally expressed as a pipeline of small computational kernels known as stencils. Halide is a popular domain-specific language and compiler designed to implement image processing algorithms. Halide uses simple language constructs to express what to compute and a separate scheduling colanguage for expressing when and where to perform the computation. This approach has demonstrated performance comparable to or better than hand-optimized code. Until now, however, Halide has been restricted to parallel shared memory execution, limiting its performance for memory-bandwidth-bound pipelines or largescale image processing tasks.

We present an extension to Halide to support distributedmemory parallel execution of complex stencil pipelines. These extensions compose with the existing scheduling constructs in Halide, allowing expression of complex computation and communication strategies. Existing Halide applications can be distributed with minimal changes, allowing programmers to explore the tradeoff between recomputation and communication with little effort. Approximately 10 new of lines code are needed even for a 200 line, 99 stage application. On nine image processing benchmarks, our extensions give up to a $1.4 \times$ speedup on a single node over regular multithreaded execution with the same number of cores, by mitigating the effects of non-uniform memory access. The distributed benchmarks achieve up to $18 \times$ speedup on a 16 node testing machine and up to $57 \times$ speedup on 64 nodes of the NERSC Cori supercomputer.
\end{abstract}

Categories and Subject Descriptors D.3.2 [Programming Languages]: Distributed and Parallel Languages

Keywords Distributed memory; Image processing; Stencils

\section{Introduction}

High-throughput and low-latency image processing algorithms are of increasing importance due to their wide applicability in fields such as computer graphics and vision, scientific and medical visualization, and consumer photography. The resolution and framerate of images that must be processed is rapidly increasing with the improvement of camera technology and the falling cost of storage space. For example, the Digitized Sky Survey [1] is a collection of several thousand images of the night sky, ranging in resolution from $14,000 \times 14,000$ to $23,040 \times 23,040$ pixels, or 200 to

Permission to make digital or hard copies of part or all of this work for personal or classroom use is granted without fee provided that copies are not made or distributed for profit or commercial advantage and that copies bear this notice and

PPoPP '16, March 12-16, 2016, Barcelona, Spain

ACM $978-1-4503-4092-2 / 16 / 03$

http://dx.doi.org/10.1145/2851141.2851157

(c) $($ )

This work is licensed under a Creative Commons Attribution-NonCommercial-NoDerivs International 4.0 License
500 megapixels. Canon, a consumer-grade camera manufacturer, recently introduced a 250 megapixel image sensor [2]. Processing such large images is a non-trivial task: on modern multicore hardware, a medium-complexity filter such as a bilateral grid [12] can easily take up to 10 seconds for a 500 megapixel image.

Halide [29], a domain-specific language for stencil pipelines, is a popular high-performance image processing language, used in Google+ Photos, and the Android and Glass platforms [28]. A major advantage of Halide is that it separates what is being computed (the algorithm) from how it is computed (the schedule), enabling programmers to write the algorithm once in a high-level language, and then quickly try different strategies to find a highperforming schedule. Halide code often outperforms hand-written expert-optimized implementations of the same algorithm.

When many stencils are composed into deep pipelines such as the local Laplacian filter [27], the inter-stage data dependencies easily become very complex, as visualized in Figure 1. In the case of a local Laplacian filter, many different resampling, data-dependent gathers and horizontal and vertical stencils combine to create a complex network of dependencies. Such complexity makes rewriting a program to experiment with different optimization strategies for computation extremely time consuming. A programmer can easily explore this space with Halide's separation of algorithm and schedule.

Halide is currently limited to shared-memory execution. For the common case where an image processing pipeline is memorybandwidth bound, the performance ceiling of these tasks is solely determined by the memory bandwidth of the executing system. Adding additional parallelism with more threads for such pipelines therefore does not result in higher performance. And, with modern multi-socket platforms embracing a non-uniform memory access (NUMA) architecture, simplistic parallel scheduling such as the work queue used by Halide often achieves poor parallel efficiency due to frequent misses into remote memory or cache. When processing today's images of 100s of megapixels, shared-memory execution limits potential performance.

We address this challenge with our distributed language and compiler extensions. By augmenting Halide programs with the ability to seamlessly distribute data and execution across many compute nodes, we offer the ability to overcome the limitations of shared-memory pipelines with very little effort. Distributed Halide pipelines gain access to more parallelism and increased memory bandwidth, and exhibit better hardware utilization of each individual node. The language extensions for distribution fit well within the existing scheduling language constructs, which can still be used to explore schedules for the on-node computation. The ability to use the a single scheduling language for both distribution and onnode scheduling is important, since depending on the distribution strategy, different on-node schedules yield the best overall performance. 


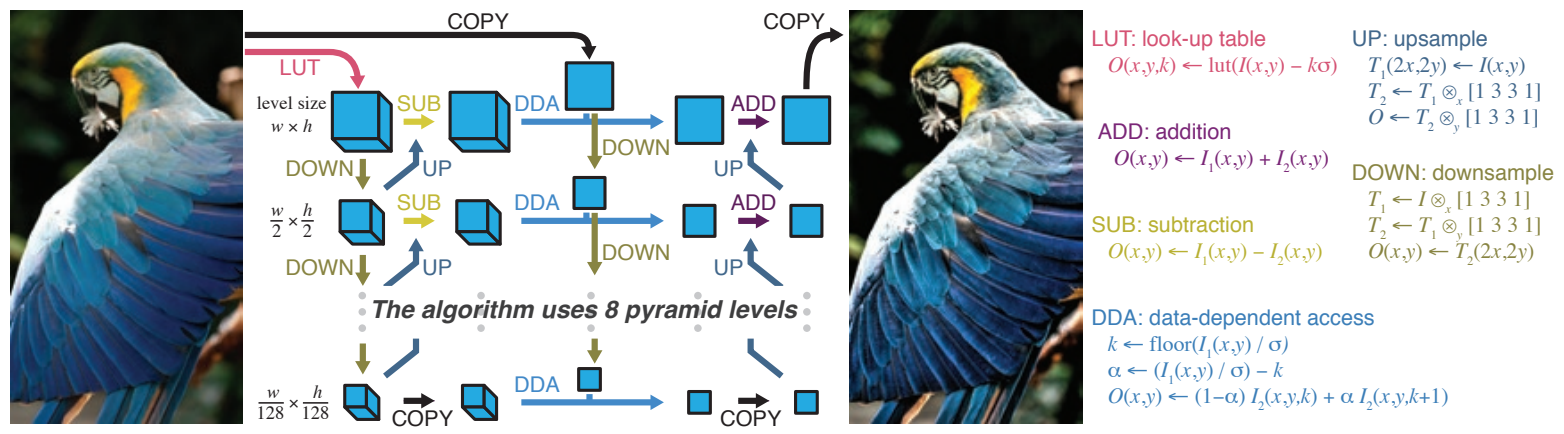

Figure 1: Data dependencies in a local Laplacian filter. Each box represents intermediate data, and arrows represent functions (color-coded with their bodies on the right) defining the data. Image reproduced with permission from [29].

\subsection{Contributions}

In this paper, we present language extensions for distributing pipelines and a new compiler backend that generates distributed code. In particular, the contributions of this paper are:

- A Halide language extension for distributing image processing pipelines, requiring programmers to write only approximately 10 new lines of code. We show that it is possible to describe complex organizations of data and computation both on-node and across multiple machines using a single, unified scheduling language.

- The implementation of a Halide compiler backend generating distributed code via calls to an MPI library.

- A demonstration of how distributed pipelines can achieve a $1.4 \times$ speedup on a single node with the same number of cores over regular multithreaded execution by mitigating NUMA effects.

- Evaluation of nine distributed image processing benchmarks scaling up to 2,048 cores, with an exploration of redundant work versus communication tradeoff.

The rest of this paper is organized as follows. Section 2 summarizes the necessary background on Halide including simple scheduling semantics. Section 3 introduces the new distributed scheduling directives. Section 4 discusses distributed code generation and Section 5 evaluates distributed Halide on several image processing benchmarks. Section 6 discusses related work, and Section 7 concludes.

\section{Halide Background}

Halide [29] is a domain-specific language embedded in C++ for image processing. One of its main points of novelty is the fundamental, language-level separation between the algorithm and schedule for a given image processing pipeline. The algorithm specifies what is being computed, and the schedule specifies how the computation takes place. By separating these concerns in the language, a programmer only writes an algorithm once. When hardware requirements change, or new features such as larger vector registers or larger caches become available, the programmer must only modify the schedule to take advantage of them.

As a concrete example, consider a simple $3 \times 3$ box blur. One typical method to compute this is a 9-point stencil, computing an output pixel as the average value of the neighboring input pixels. In Halide, such a blur can be expressed in two stages: first a horizontal blur over a $1 \times 3$ region of input pixels, followed by a vertical $3 \times 1$ blur. This defines the algorithm of the blur, and is expressed in Halide as:

$\operatorname{bh}(x, y)=($ in $(x-1, y)+$ in $(x, y)+$ in $(x+1, y)) / 3$; $b v(x, y)=(b h(x, y-1)+b h(x, y)+b h(x, y+1)) / 3$;

where each output pixel output $(x, y)=b v(x, y)$.

Halide schedules express how a pipeline executes. For example, one natural schedule computes the entire bh horizontal blur stage before computing the bv stage. In Halide's scheduling language, this is known as computing the function at "root" level, and is expressed as:

bh.compute_root ();

With this schedule, our blur pipeline is compiled to the following pseudo-code of memory allocations and loop nests:

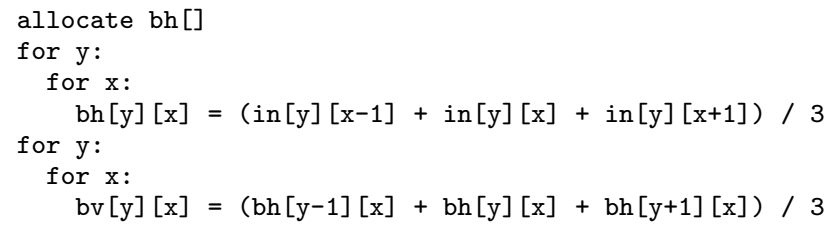

By opting to completely compute the horizontal blur before beginning the vertical blur, we schedule the pipeline such that there is no redundant computation: each intermediate pixel in bh is only calculated once. However, this sacrifices temporal locality. A pixel stored into the bh temporary buffer may not be available in higher levels of the memory hierarchy by the time it is needed to compute bv. This time difference is known as reuse distance: a low reuse distance is equivalent to good temporal locality.

Because the horizontal blur is so cheap to compute, a better schedule may compute pixels of bh multiple times to improve reuse distance. One possibility is to compute a subset of the horizontal blur for every row of the vertical blur. Because a single row of the vertical blur requires three rows in bh (the row itself and the rows above and below), we can compute only those three rows of bh in each iteration of bv . y. In Halide, the schedule:

bh.compute_at (bv, y);

is lowered to the loop nest:

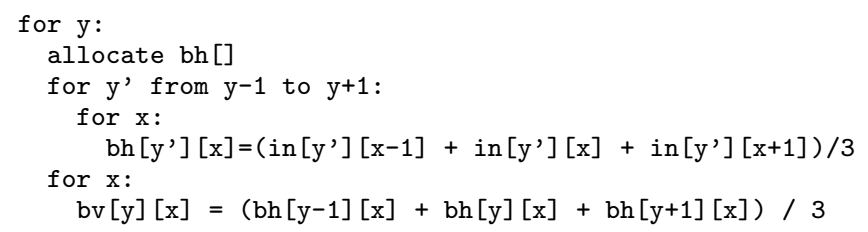


Note that the inner iteration of y' ranges from $y-1$ to $y+1$ : the inner loop nest is calculating the currently needed three rows of bh. Some details such as buffer indexing and how the Halide compiler determines loop bounds have been elided here for clarity, but the essence is the same. The tradeoff explored by these two schedules is that of recomputation versus locality. In the first schedule, there is no recomputation, but locality suffers, while in the second schedule, locality improves, as values in bh are computed soon before they are needed, but we redundantly compute rows of bh.

Halide also offers a simple mechanism for adding parallelism to pipelines on a per-stage per-dimension basis. To compute rows of output pixels in parallel, the schedule becomes:

bh.compute_at (bv, y);

bv.parallel (y);

which is lowered to the same loop nest with the outermost bv $y$ loop now a parallel for loop. The runtime mechanism for supporting parallel for loops encapsulates iterations of the parallel dimension $\mathrm{bv} \cdot \mathrm{y}$ into function calls with a parameter specifying the value of bv. $y$ to be computed. Then, a work queue of all iterations of bv. $y$ is created. A runtime thread pool (implemented using e.g. pthreads) pulls iterations from the work queue, executing iterations in parallel. This dynamic scheduling of parallelism can be a source of cache inefficiencies or NUMA effects, as we explore in Section 5.

Designing good schedules in general is a non-trivial task. For blur the most efficient schedule we have found, and the one we compare against in our evaluation is:

bh.store_at (bv, y).compute_at (bv, yi).vectorize $(x, 8)$; bv.split(y, y, yi, 8).vectorize (x, 8).parallel(y);

For large pipelines such as the local Laplacian benchmark consisting of approximately 100 stages, the space of possible schedules is enormous. One solution to this problem is applying an autotuning system to automatically search for efficient schedules, as was explored in [29]. Other more recent autotuning systems such as OpenTuner [9] could also be applied to the same end.

\section{Distributed Scheduling}

One of the major benefits of the algorithm-plus-schedule approach taken by Halide is the ability to quickly experiment to find an efficient schedule for a particular pipeline. In keeping with this philosophy, we designed our distributed Halide language extensions to be powerful enough to express complex computation and communication schemes, but simple enough to require very little effort to find a high-performance distributed schedule.

There are many possible language-level approaches to express data and computation distributions. We strove to ensure that the new scheduling constructs would compose well with the existing language both in technical (i.e. the existing compiler should not need extensive modification) and usability terms. The new extensions needed to be simple enough for programmers to easily grasp but powerful enough to express major tradeoffs present in distributed-memory programs.

Striking a balance between these two points was accomplished by adding two new scheduling directives, distribute() and compute_rank(), as well as a new DistributedImage buffer type that uses a simple syntax to specify data distributions. We show that these extensions are simple to understand, compose with the existing language, and allow complex pipelines to be scheduled for excellent scalability.

\subsection{Data Distribution via DistributedImage}

Input and output buffers in Halide are represented using the userfacing Image type. Images are multidimensional Cartesian grids of pixels and support simple methods to access and modify pixel values at given coordinates. In distributed Halide, we implemented a user-facing DistributedImage buffer type which supports additional methods to specify the distribution of data to reside in the buffer.

A DistributedImage is declared by the user with the dimensions' global extents and names. A data distribution for each DistributedImage is specified by the user by using the placement () method, which returns an object supporting a subset of the scheduling directives used for scheduling Halide functions, including the new distribute() directive. "Scheduling" the placement specifies a data distribution for that image. The following example declares a DistributedImage with global extents width and height but distributed along the y dimension.

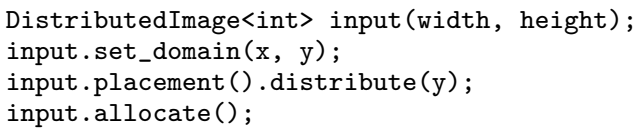

It is important to note that the amount of backing memory allocated on each rank with the allocate() call is only the amount of the per-rank size of the image. The size of the local region is determined by the logic explained next in Section 3.2. The call to allocate () must occur separately and after all scheduling has been done via placement () in order to calculate the per-rank size.

For a rank to initialize its input image, the DistributedImage type supports conversion of local buffer coordinates to global coordinates and vice versa. This design supports flexible physical data distributions. For example, if a monolithic input image is globally available on a distributed file system, each rank can read only its local portion from the distributed file system by using the global coordinates of the local region. Or, if input data is generated algorithmically, each rank can initialize its data independently using local or global coordinates as needed. In either case, at no point does an individual rank allocate or initialize the entire global image. Output data distribution is specified in exactly the same manner.

\subsection{Computation Distribution}

To support scheduling of distributed computations, we introduce two new scheduling directives: distribute() and compute_rank().

The distribute() directive is applied to dimensions of individual pipeline stages, meaning each stage may be distributed independently of other stages. Because dimensions in Halide correspond directly to levels in a loop nest, a distributed dimension corresponds to a distributed loop in the final loop nest. The iteration space of a distributed loop dimension is split into slices according to a block distribution. Each rank is responsible for exactly one contiguous slice of iterations of the original loop dimension.

The compute_rank () directive is applied to an entire pipeline stage, specifying that the computed region of the stage is the region required by all of its consumers on the local rank (we adopt the MPI terminology "rank" to mean the ID of a distributed process). Scheduling a pipeline stage with compute_rank() ensures that locally there will be no redundant computation, similar to the semantics of compute_root () in existing Halide. However, different ranks may redundantly compute some regions of compute_rank functions. Therefore, compute_rank () allows the expression of globally redundant but locally nonredundant computation, a new point in the Halide scheduling tradeoff space.

The block distribution is defined as follows. Let $R$ be the number of MPI processes or ranks available and let $w$ be the global extent of a loop dimension being distributed. Then the slice size $s=\lceil w / R\rceil$, and each rank $r$ is responsible for iterations

$$
[r s, \min (w,(r+1) s))
$$




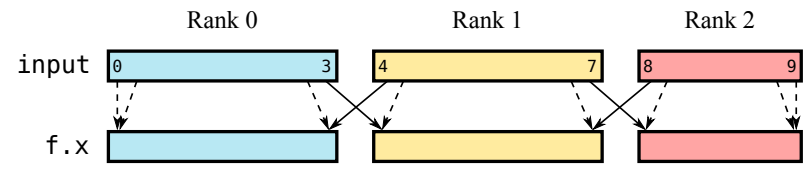

Figure 2: Communication for 1D blur. Dotted lines represent onnode access, solid lines represent communication.

where $[u, v)$ denotes the half-open interval from $u$ to $v$. This has the effect of assigning the last rank fewer iterations in the event $R$ does not evenly divide $w$.

The slicing of the iteration space is parameterized by the total number of ranks $R$ and the current rank $r$. Our code generation (explained in Section 4) uses symbolic values for these parameters; thus, running a distributed Halide pipeline on different numbers of ranks does not require recompiling the pipeline.

The distribute () directive can also be applied to two or three dimensions of a function to specify multidimensional (or nested) distribution. For nested distribution, the user must specify the size of the desired processor grid to distribute over: we currently do not support parametric nested distributions as in the one dimensional case. The nested block distribution is defined as follows. Let $x$ and $y$ respectively be the inner and outer dimensions in a 2D nested distribution, let $w$ and $h$ be their respective global extents and let $a$ and $b$ be the respective extents of the specified processor grid. Then the slice sizes are $s_{x}=\lceil w / a\rceil$ and $s_{y}=\lceil h / b\rceil$. Each rank $r$ is responsible for a $2 \mathrm{D}$ section of the original iteration space, namely:

$$
\begin{aligned}
& x \in\left[r(\bmod a) s_{x}, \min \left(w,(r(\bmod a)+1) s_{x}\right)\right) \\
& y \in\left[(r \backslash a) s_{y}, \min \left(h,(r \backslash a+1) s_{y}\right)\right)
\end{aligned}
$$

where $u \backslash v$ denotes integer division of $u$ and $v$. For 3D distribution, letting $d$ be the extent of the third dimension $z, c$ be the third extent of the specified processor grid, $s_{z}=\lceil d / c\rceil$, the iteration space for rank $r$ is:

$$
\begin{aligned}
x \in & {\left[(r(\bmod a b))(\bmod a) s_{x},\right.} \\
& \left.\min \left(w,((r(\bmod a b))(\bmod a)+1) s_{x}\right)\right) \\
y \in & {\left[((r(\bmod a b)) \backslash a) s_{y},\right.} \\
& \left.\min \left(h,(((r(\bmod a b)) \backslash a)+1) s_{y}\right)\right) \\
z \in & {\left[(r \backslash(a b)) s_{z}, \min \left(d,(r \backslash(a b)+1) s_{z}\right)\right) . }
\end{aligned}
$$

\subsection{Introductory Example}

As an example, consider a simple one-dimensional blur operation:

$f(x)=(\operatorname{input}(x-1)+\operatorname{input}(x+1)) / 2 ;$

We can distribute this single-stage pipeline using the two new language features (eliding the boilerplate set_domain() and allocate () calls):

DistributedImage input(width);

input.placement ().distribute (x);

f.distribute $(x)$;

With this schedule, the input buffer is distributed along the same dimension as its consumer stage $f$.

We call the slice of the input buffer residing on each rank the owned region. Because $f$ is a stencil, we must also take into account the fact that to compute an output pixel $f(x)$ requires input $(x-1)$ and input $(x+1)$. We denote this the required region of buffer input.

Suppose that the width of input is 10 pixels, and we have 3 ranks to distribute across. Then the following table enumerates each rank's owned and required regions of input, according to the distributed schedule:

\begin{tabular}{rccc} 
& Rank 0 & Rank 1 & Rank 2 \\
\cline { 2 - 4 } f.x & $0-3$ & $4-7$ & $8-9$ \\
input owned & $0-3$ & $4-7$ & $8-9$ \\
input required & $0-4$ & $3-8$ & $7-9$
\end{tabular}

Because the input buffer is distributed independently from its consumer, and distributed slices are always disjoint by construction, the required region is larger than the owned region for buffer input. Therefore, the required values of input will be communicated from the rank that owns them. In this example, rank 1 will send input (4) to rank 0 and input (7) to rank 2 (and receive from both ranks 0 and 2 as well). The communication is illustrated in Figure 2.

The region required but not owned is usually termed the ghost zone (see e.g. [22]), and the process of exchanging data is called border exchange or boundary exchange. In distributed Halide, ghost zones are automatically inferred by the compiler based on the schedule, and the communication code is inserted automatically. The mechanics of how the ghost zones are calculated and communicated are detailed in Section 4.

This example describes a single-stage pipeline, but for multistage pipelines, data must be communicated between the stages. Between each distributed producer and consumer, ghost zones are determined and communication code is automatically inserted by the compiler, just as the case above of communicating input to the ranks computing $f$.

\subsection{Recomputation versus Communication}

A fundamental tradeoff exposed by the new distribute() and compute_rank() scheduling directives is recomputation versus communication. In some cases, it may be advantageous to locally recompute data in the ghost zone, instead of communicating it explicitly from the rank that owns the data. While there are models of distributed computation and communication (e.g. [7]) that can be applied to make one choice over the other, these models are necessarily approximations of the real world. For optimal performance, this choice should be made empirically on a per-application basis. With distributed Halide, we can explore the tradeoff not just per application, but per stage in an image processing pipeline.

In distributed Halide, there are three points on the spectrum of this tradeoff. Globally and locally non-redundant work is expressed by the compute_root ()+distribute () directives, ensuring the function is computed exactly once by a single rank for a given point in the function's domain. This is the typical case, but communication may be required for consumers of a pipeline stage distributed in this manner. Globally redundant but locally non-redundant work is expressed by the new compute_rank() directive, meaning a given point in the function domain may be computed by multiple ranks, but on each rank it is only ever computed once. No communication is required in this case, as each rank computes all of the data it will need for the function's consumers. Finally, globally and locally redundant work is expressed by scheduling a function compute_at inside of its distributed consumer. A given point in the function domain may be computed multiple times within a single rank as well as across ranks, but no communication is required.

No one point on this tradeoff spectrum is most efficient for all applications. Distributed Halide exposes this tradeoff to the user, allowing the best choice to be made on a case-by-case basis.

Recalling the $3 \times 3$ box blur example from Section 2, this distributed schedule expresses globally and locally redundant computation of bh:

bh.compute_at (bv, y); bv.parallel(y).distribute(y);

Crucially, even though the pipeline is distributed, there is no communication of bh, but instead each rank computes the region of bh 


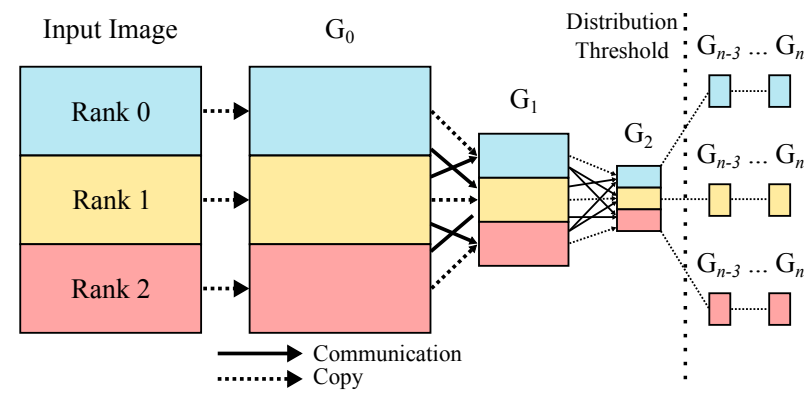

Figure 3: Communication for the Gaussian pyramid computation in the Local Laplacian benchmark. The final three levels after the "distribution threshold" are redundantly computed by every rank.

it needs for each of the rows it produces in bv. Because the computation of bh occurs within the distributed dimension bv $\mathrm{y}$, each rank computes bh separately. This means the overlapping rows of $\mathrm{bh}$ that are required for each iteration of bv $\cdot \mathrm{y}$ are computed redundantly and locally. Communication is still required for the region of the input buffer needed to compute the local portion of bv, but no communication is required for the bh stage.

An alternative schedule that contains no redundant computation, but requires communication of both the input buffer and the bh stage is:

bh.compute_root(). distribute(y);

bv.parallel (y).distribute(y);

The horizontal blur is computed entirely before the vertical blur begins. Before computing bh, the ghost zone data (required but not owned data) of the input buffer must be communicated between ranks. Then, the overlapping rows of bh. $\mathrm{y}$ in the ghost zone must be communicated before computation of bv can begin. This is a case where there is no redundant computation (all pixels of bh are computed exactly once globally).

A more extreme version of redundant computation is the following:

bh.compute_root ();

bv. parallel (y).distribute (y);

With this schedule, the entire horizontal blur (i.e. over the entire global input image) is evaluated on every rank. This is wasteful in terms of computation, as each rank will compute more of bh than it needs. However, locally there is no redundant computation, and globally there is no communication required for bh. Using this particular strategy is crucial for scalability on the local Laplacian benchmark, as shown in Figure 3. The final three stages of the image pyramid in Figure 3 are recomputed on every node, as the data is small enough that recomputation is faster than communication.

Finally, this schedule express globally redundant but locally non-redundant computation:

bh.compute_rank();

bv . parallel (y).distribute (y);

Each rank will non-redundantly (i.e. at root level on each rank) compute only the region of bh required to compute the local portion of bv. No communication is required in this case. However, neighboring ranks will compute overlapping rows of bh, meaning globally there is some redundant computation.

\subsection{On-Node Scheduling}

The distribute() and compute_rank() directives compose with existing Halide scheduling directives. All ranks inherit the same schedule for computing the local portion of a global compu- tation. To express the common idiom of distributing data across ranks and locally parallelizing the computation on each rank, the parallel() directive can be used in conjunction with the distribute() directive.The various existing scheduling directives can be composed arbitrarily to arrive at complex schedules of computation and communication. For example, the following schedule (taken from the block transpose benchmark) causes $f$ to be computed by locally splitting the image into $16 \times 16$ tiles, vectorizing and unrolling the computation of each tile, distributing the rows of $f$ and locally parallelizing over the rows of tiles:

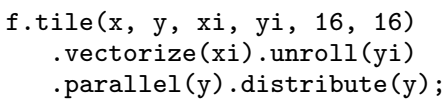

The flexibility of this approach allows programmers to specify efficient on-node schedules and freely distribute the computation of each stage, without worrying about calculating ghost zones or writing communication code.

\subsection{Limitations}

The current implementation of distributed Halide supports all of the features described above, but there are several engineering challenges remaining to be addressed. In particular, ahead of time compilation has not yet been implemented; currently only JITted pipelines can be distributed. Only dimensions that can be parallelized can be distributed, i.e. distributing data-dependent dimensions (as used in a histogram, for example) is unimplemented. Nested distributions with a parametric processor grid are not yet supported, as previously mentioned in Section 3.2. Any stage writing to a distributed output buffer must currently have the same distribution as the output buffer. Reordering storage with the reorder_storage () scheduling directive is not yet supported for distributed dimensions.

\section{Code Generation}

This section details the new analysis and lowering pass added to the Halide compiler to generate code for distributed pipelines. There are two components of code generation: determining ghost zones for each distributed producer-consumer relationship in the pipeline, and inserting MPI calls to communicate required data in the ghost zones. The mechanisms presented here are similar to those in previous work such as [15] for affine loop nests; see Section 6 for a discussion of their work. The main advantage of our approach lies in the simplicity of ghost zone inference and code generation.

\subsection{Ghost Zone Inference}

Our ghost zone inference relies heavily on the axis-aligned bounding box bounds inference already present in the Halide compiler. In regular Halide, bounds information is needed to determine allocation sizes of intermediate temporary memory, iteration spaces of pipeline stages and out-of-bounds checks, among other things.

For each consumer stage in the pipeline, we generate the owned and required information for its inputs, which may be DistributedImages or earlier stages in the pipeline. We derive the owned and required regions in terms of the global buffer bounds the programmer provides via the DistributedImage type.

Recall the iteration space slicing from Section 3.2 and the onedimensional blur $\mathrm{f}$ from Section 3.3. Applying bounds inference to the distributed loop nest generates the symbolic owned and required regions for computing $f$ (omitting boundary conditions for simplicity): 


\begin{tabular}{rc} 
& Rank $r$ \\
\hline f.x & $r s$ to $(r+1) s$ \\
input owned & $r s$ to $(r+1) s$ \\
input required & $r s-1$ to $(r+1) s+1$
\end{tabular}

Identical inference is done for every producer-consumer relationship in the distributed pipeline. Multidimensional buffers are handled with the same code: the bounds inference information generalizes across arbitrary dimensions.

Because these regions are parameterized by a rank variable, any particular rank $r$ can determine the owned and required regions for another rank $r^{\prime}$. A global mapping of rank to owned region is never explicitly computed or stored; instead it is computed dynamically when needed. By computing data locations lazily, we avoid creating and accessing a mapping of global problem space to compute rank.

Border exchange is required for the distributed inputs of each consumer in the pipeline. The inputs could be DistributedImages or earlier distributed stages in the pipeline. In the following discussion we will refer to these collectively as "data sources," as the owned and required regions are computed the same way no matter the type of input.

To perform a border exchange for data source $b$ between two ranks $r$ and $r^{\prime}$, each must send data it owns to the other rank if it requires it. Let $H(b ; r)$ be the owned (or "have") region of data source $b$ by rank $r$ and let $N(b ; r)$ be the required (or "need") region. If $H(b ; r)$ intersects $N\left(b ; r^{\prime}\right)$, rank $r$ owns data required by $r^{\prime}$, and symmetric send/receive calls must be made on each rank.

The $H$ and $N$ regions are represented by multidimensional axisaligned bounding boxes, where each dimension has a minimum and maximum value. Computing the intersection $I$ of the two bounding boxes is done using the following equations:

$$
\begin{aligned}
& I_{d}\left(b, r, r^{\prime}\right) \cdot \min =\max \left\{H_{d}(b ; r) \cdot \min , N_{d}\left(b ; r^{\prime}\right) \cdot \min \right\} \\
& I_{d}\left(b, r, r^{\prime}\right) \cdot \max =\min \left\{H_{d}(b ; r) \cdot \max , N_{d}\left(b ; r^{\prime}\right) \cdot \max \right\}
\end{aligned}
$$

where $B_{d}(\cdot) \cdot \min / \max$ denotes the minimum or maximum in dimension $d$ of bounding box $B$. We generate the communication code using this static representation of the intersection between what a rank $r$ has and what a rank $r^{\prime}$ needs.

The intersections are calculated using global coordinates (relative to the global input/output buffer extents). The actual buffers allocated on each rank are only the size of the local region. Thus, before passing the intersection regions to the MPI library, they must be converted to local coordinates, offset from 0 instead of $r s$. For rank $r$ and a region $X$ of data source $b$ in global coordinates, we define $L(X, b ; r)$ to be $X$ in coordinates local to rank $r$. $L$ is computed by subtracting the global minimum from the global offset to compute a local offset:

$$
\begin{aligned}
& L_{d}(X, b ; r) \cdot \min =X_{d} \cdot \min -H_{d}(b ; r) \cdot \min \\
& L_{d}(X, b ; r) \cdot \max =X_{d} \cdot \max -H_{d}(b ; r) \cdot \min .
\end{aligned}
$$

\subsection{Communication Code Generation}

Preceding each consumer stage in the pipeline, we inject MPI calls to communicate the ghost zone region of all distributed inputs required by the stage. This process is called the border exchange.

Generating the code to perform border exchange uses the inferred symbolic ghost zone information. For a data source b, the function border_exchange(b) is generated with the following body:

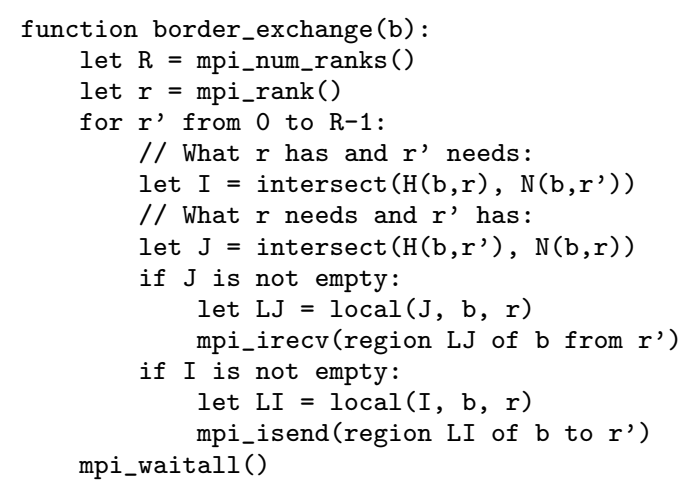

The number of ranks $R$ and the current rank $r$ are symbolic values determined at runtime by calls to MPI. Keeping these values symbolic means that the bounds inference applied to this loop nest will be in terms of these symbols. This allows us to not require recompilation when changing the number of ranks.

A call to the border exchange is inserted preceding the computation of a stage. Recall the distributed one-dimensional blur from Section 3.3. As was illustrated, the owned region of buffer input is smaller than the required region, necessitating border exchange on the input buffer:

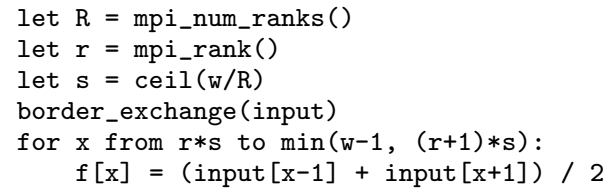

Performing border exchanges for multidimensional buffers requires more specialized handling. In particular, the regions of multidimensional buffers being communicated may not be contiguous in memory. We handle this case using MPI derived datatypes, which allow a non-contiguous region of memory to be sent or received with a single MPI call. The MPI implementation automatically performs the packing and unpacking of the non-contiguous region, as well as allocation of scratch memory as needed. This, along with wide support on a variety of popular distributed architectures, was among the reasons we chose to use MPI as the target of our code generation.

\section{Evaluation}

We evaluate distributed Halide by taking nine existing image processing benchmarks written in Halide and distributing them using the new scheduling directives and the DistributedImage type. The benchmarks range in complexity from the simple two-stage box blur to an implementation of a local Laplacian filter with 99 stages. For each, we begin with the single-node schedule tuned by the author of the benchmark. All schedules are parallelized, and usually use some combination of vectorization, loop unrolling, tiling and various other optimizations to achieve excellent singlenode performance. We then modify the schedules to use the distributed Halide extensions. Many of the benchmarks are the same as in the original Halide work [29]. A brief description of each benchmark follows.

Bilateral grid This filter blurs the input image while preserving edges [12]. It consists of a sequence of three-dimensional blurs over each dimension of the input image, a histogram calculation and a linear interpolation over the blurred results.

Blur An implementation of the simple 2D 9-point box blur filter.

Camera pipe An implementation of a pipeline used in digital cameras, which transforms the raw data collected by the image 


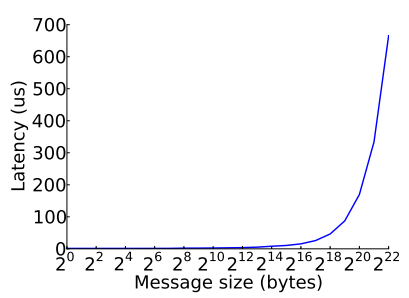

(a) Point-to-point latency

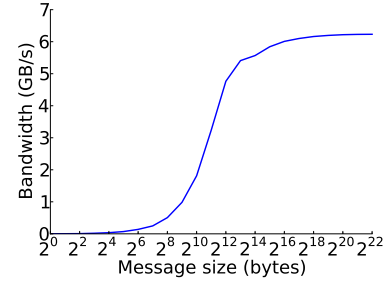

(b) Point-to-point bandwidth
Figure 4: Network point-to-point latency and bandwidth measurements for our testing environment.

sensors into a usable digital image. This pipeline contains more than 20 interleaved stages of interpolation, de-mosaicing, and color correction, and transforms a two-dimensional input into a threedimensional output image.

Interpolate A multi-scale image pyramid [6] is used to interpolate an input image at many different resolutions. The image pyramid consists of 10 levels of inter-dependent upsampling and downsampling and interpolation between the two.

Local Laplacian This filter [27] performs a complex edgepreserving contrast and tone adjustment using an image pyramid approach. The pipeline contains 99 stages and consists of multiple 10-level image pyramids including a Gaussian pyramid and Laplacian pyramid. Between each stage are complex and data-dependent interactions, leading to an incredibly large schedule space. This is our most complex benchmark.

Resize This filter implements a $130 \%$ resize of the input image using cubic interpolation, consisting of two separable stages.

Sobel An implementation of image edge-detection using the well-known Sobel kernel.

Transpose An implementation of a blocked image transpose algorithm.

Wavelet A Daubechies wavelet computation.

Our testing environment is a 16 node Intel Xeon E5-2695 v2 @ 2.40GHz Infiniband cluster with Ubuntu Linux 14.04 and kernel version 3.13.0-53. Each node has two sockets, and each socket has 12 cores, for a total of 384 cores. Hyperthreading is enabled, and the Halide parallel runtime is configured to use as many worker threads as logical cores. The network topology is fully connected.

To analyze the network performance of our test machine we ran the Ohio State microbenchmark suite [26]. The point-to-point MPI latency and effective bandwidth measurements are reported in Figures $4 \mathrm{a}$ and $4 \mathrm{~b}$ respectively.

Due to the presence of hyperthreading and the dynamic load balancing performed by the Halide parallel runtime, the performance numbers had non-negligible noise. As the input size decreases for a multithreaded Halide pipeline, the variance in runtime increases. For a $1000 \times 1000$ parallel blur with unmodified Halide, over 1,000 iterations we recorded a standard deviation of $21.3 \%$ of the arithmetic mean runtime. At $23,000 \times 23,000$, we recorded a standard deviation of $2.1 \%$. In a distributed pipeline, even though the global input may be large enough to lower the variance, each rank has a smaller region of input and therefore higher variance. To mitigate this variance as much as possible in our measurements of distributed Halide, we take median values of 50 iterations across for each node and report the maximum recorded median. The timing results were taken using

\begin{tabular}{cccc} 
Input Size & $\begin{array}{c}\text { Distr. Halide } \\
(\mathrm{s})\end{array}$ & $\begin{array}{c}\text { OpenCV } \\
(\mathrm{s})\end{array}$ & Speedup \\
\hline $1000 \times 1000$ & 0.002 & 0.002 & $1.0 \times$ \\
$2000 \times 2000$ & 0.002 & 0.009 & $1.255 \times$ \\
$4000 \times 4000$ & 0.004 & 0.033 & $8.252 \times$ \\
$10000 \times 10000$ & 0.023 & 0.223 & $9.697 \times$ \\
$20000 \times 20000$ & 0.096 & 0.917 & $9.552 \times$ \\
$50000 \times 50000$ & 0.688 & 5.895 & $8.568 \times$
\end{tabular}

Table 1: Speedup of Distributed Halide box blur over OpenCV.

\begin{tabular}{cccc} 
Input Size & $\begin{array}{c}\text { Distr. Halide } \\
(\mathrm{s})\end{array}$ & $\begin{array}{c}\text { OpenCV } \\
(\mathrm{s})\end{array}$ & Speedup \\
\hline $1000 \times 1000$ & 0.003 & 0.004 & $1.020 \times$ \\
$2000 \times 2000$ & 0.004 & 0.019 & $4.752 \times$ \\
$4000 \times 4000$ & 0.010 & 0.074 & $7.4 \times$ \\
$10000 \times 10000$ & 0.054 & 0.446 & $8.259 \times$ \\
$20000 \times 20000$ & 0.183 & 1.814 & $9.913 \times$ \\
$50000 \times 50000$ & 1.152 & 12.674 & $11.001 \times$
\end{tabular}

Table 2: Speedup of Distributed Halide Sobel edge detection over OpenCV.

clock_gettime (CLOCK_MONOTONIC), a timer available on Linux with nanosecond resolution.

We first compare two benchmarks to popular open-source optimized versions to illustrate the utility of parallelizing these benchmarks, even at small input sizes. We then report performance of distributed Halide in two categories: scaling and on-node speedup.

\subsection{OpenCV Comparison}

To establish the utility of using Halide to parallelize and distribute these benchmarks, we first compare against reference sequential implementations of the box blur and edge-detection benchmarks. We chose to compare against OpenCV [4], a widely-used open source collection of many classical and contemporary computer vision and image processing algorithms. The OpenCV implementations have been hand-optimized by experts over the almost 20 years it has been in development.

We chose box blur and edge-detection to compare because OpenCV contains optimized serial implementations of both kernels, whereas both Halide benchmarks are fully parallelized. We built OpenCV on our test machine using the highest vectorization settings (AVX) defined by its build system. The results are summarized in Tables 1 and 2. For the largest tested input size, the parallel single-node Halide implementation was $8.5 \times$ faster for box blur and $11 \times$ faster for Sobel. Even for these simple pipelines there is a need for parallelism.

\subsection{Scaling}

To test the scalability of distributed Halide, we ran each benchmark on increasing numbers of ranks with a fixed input size. We repeated the scaling experiments with several input sizes up to a maximum value. These results measure the benefit of distributed computation when the communication overhead is outstripped by the performance gained from increased parallelism. As a baseline, for each benchmark and input size, we ran the non-distributed version on a single node. As mentioned previously, the parallel runtime was configured to use as many threads as logical cores. Therefore, for all benchmarks we normalized to the non-distributed, singlenode, 24-core/48-thread median runtime on the given input size.

When increasing the number of nodes, we adopted a strategy of allocating two ranks per node. This maximized our distributed per- 

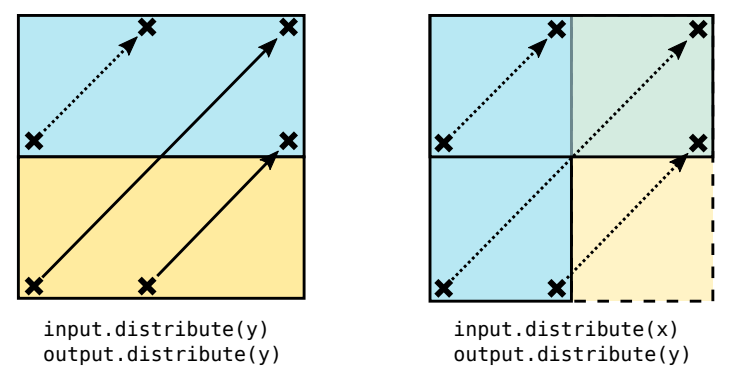

Figure 6: Two data distributions in transpose. By distributing the input along the opposite dimension as the output, only local accesses (dotted lines) are required to transpose the input, as opposed to the explicit communication (solid lines) in the other case.

\begin{tabular}{rcrr} 
\# nodes & Input Distr. & Runtime (s) & Speedup \\
\hline 1 & N/A & 0.119 & $1.0 \times$ \\
16 & $\mathrm{y}$ & 0.067 & $1.779 \times$ \\
16 & $\mathrm{x}$ & 0.007 & $16.172 \times$
\end{tabular}

Table 3: Speedup of transpose on $23000 \times 23000$ image with different input distributions.

formance by mitigating effects of the NUMA architecture, explored in more detail in the following section.

The scaling results from all nine benchmarks are presented in Figure 5. Broadly speaking, the results fall into three categories.

In the first category are the bilateral grid, blur, resize, Sobel, transpose and wavelet benchmarks. This category represents benchmarks exhibiting predictable and well-scaling results. The distribution strategy in bilateral grid, blur and wavelet was the same, utilizing multiple stages with redundant computation. The only data source requiring communication for these benchmarks was the input buffer itself: once the (relatively small) input ghost zones were received, each rank could proceed independently, maximizing parallel efficiency. On bilateral grid, even the smallest input size achieved a $8.8 \times$ speedup on 16 nodes, with the maximum input size achieving $12.1 \times$ speedup. On blur and wavelet, the smallest input size achieved a speedup only around $4 \times$ : both blur and wavelet have very low arithmetic complexity, meaning with small input sizes each rank was doing very little computation.

Transpose, which belongs to the first category, demonstrated good scaling when distributing the input and output buffers along opposite dimensions. We distributed the input along the $\mathrm{x}$ dimension and output along the y dimension, requiring only on-node data access to perform the transpose. If we distributed both the input and output along the same dimension, the ghost zone for the input on each rank would have required communication. These two strategies are visualized in Figure 6. In Table 3 we compare the speedup of transpose with 16 nodes using each data distribution. The difference between the two data distributions is an order of magnitude of speedup gained.

The second category consists of camera pipe and local Laplacian. These benchmarks exhibit less scalability than those in the first category. Both the camera pipe and local Laplacian pipelines are complex, leading to a large schedule space, and we expect that by exploring further schedules, even better scalability can be achieved. Regardless of their suboptimal schedules, local Laplacian and camera pipe achieved a $7.7 \times$ and $11.2 \times$ speedup on 16 nodes, respectively, for their largest input sizes.

The final category consists of interpolate. This benchmark displayed superlinear scaling for larger input sizes. The image-

\begin{tabular}{lll|ll|ll|ll}
\multirow{2}{*}{ Benchmark } & \multicolumn{2}{c}{$\mathbf{1 k} / \mathbf{1 N}$} & \multicolumn{2}{c}{$\mathbf{1 k} / \mathbf{1 6 N}$} & \multicolumn{2}{c}{$\mathbf{2 0 k} / \mathbf{1 N}$} & \multicolumn{2}{c}{$\mathbf{2 0 k} / \mathbf{1 6 N}$} \\
& App. & MPI\% & App. & MPI\% & App. & MPI\% & App. & MPI\% \\
\hline bilateral grid & 2.43 & 0.04 & 9.83 & 53.65 & 809 & 0.08 & 847 & 2.47 \\
blur & 0.19 & 14.16 & 5.87 & 93.46 & 16.4 & 0.82 & 28.8 & 12.93 \\
camera pipe & 0.86 & 13.24 & - & - & 78.5 & 0.42 & 140 & 28.24 \\
$\begin{array}{l}\text { interpolate } \\
\text { local lapla- }\end{array}$ & 1.27 & 22.64 & 23.6 & 68.31 & 188 & 3.64 & 250 & 44.05 \\
cian & 10.5 & 30.6 & 61.83 & 360 & 6.42 & 813 & 72.26 \\
resize & 0.6 & 7.61 & 3.96 & 71.45 & 59.3 & 1.42 & 131 & 42.09 \\
sobel & 0.13 & 12.32 & 7.16 & 92.93 & 18.6 & 1.22 & 30.7 & 23.41 \\
transpose & 0.09 & 7.58 & 1.01 & 69.66 & 9.83 & 0.10 & 14.7 & 39.84 \\
wavelet & 0.20 & 10.81 & 2.05 & 66.15 & 13 & 0.67 & 20.9 & 27.52
\end{tabular}

Table 4: Communication and computation time for each benchmark.

pyramid based interpolation displayed some of the best scaling behavior, even at a maximum input size of $20,000 \times 20,000$. We accomplished this in part by utilizing redundant computation in the later stages of the pyramid, using the strategy visualized in Figure 3. In addition, the distributed pipeline exhibits better hardware utilization: as the working set size decreases per rank, each processor can make more effective use of its cache, leading to better memory bandwidth utilization. Finally, NUMA-aware data partitioning leads to better on-node parallel efficiency, and is explored in more detail in the following subsection.

To measure an estimation of scaling efficiency, we also measured the communication overhead for each benchmark on the smallest input and a mid-range input size $(1000 \times 1000$ and $20000 \times 20000$ respectively), on a single node (with two MPI ranks) and 16 nodes (with 32 MPI ranks, one per socket). We used the MPIP [32] library to gather these results over all iterations. The results are summarized in Table 4 . The " $1 \mathrm{k} / 1 \mathrm{~N}$ " column refers to the input size of 1,000 on 1 node; similarly for the other columns. The "App" and "MPI\%" columns refer to the aggregate application execution time in seconds and the percentage of that time spent in the MPI library. Roughly speaking, the benchmarks which exhibit poor scaling (the second category) have a larger fraction of their execution time consumed by communication; for example, nearly $75 \%$ of the execution time of local Laplacian across 16 nodes is spent communicating or otherwise in the MPI library. This indicates that the distributed schedules for these benchmarks are not ideal. Further experimentation with distributed scheduling would likely lead to improved scalability on these benchmarks. We were unable to run the $1 \mathrm{k} / 16 \mathrm{~N}$ case for camera pipe because this benchmark contains a distributed dimension of only extent 30 with an input size of 1,000, which cannot be distributed across 32 ranks.

\subsection{On-Node Speedup from NUMA-Aware Distribution}

To quantify the portion of speedup seen with "distributing" a Halide pipeline on a single node, we used the open-source Intel PMU profiling tools [5]. For this experiment, we ran a 23,000 $\times 23,000$ 2D box blur under different NUMA configurations. During each run we gathered several performance counters for LLC (last level cache) misses satisfied by relevant sources. In particular we looked at:

- LLC demand read misses, any resolution (offcore_response_demand_data_rd_llc_miss_any_response)

- LLC demand read misses resolved by local DRAM (offcore_response_demand_data_rd_llc_miss_local_dram)

- LLC demand read misses resolved by remote DRAM (offcore_response_demand_data_rd_llc_miss_remote_dram)

Demand misses in this context refer to misses that were not generated by the prefetcher. We also measured misses resolved by hits in 


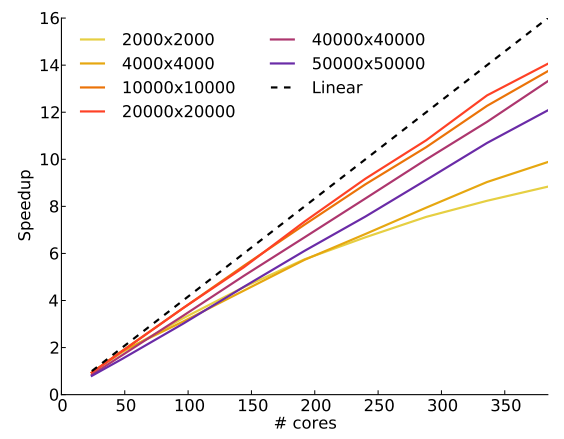

(a) Bilateral grid

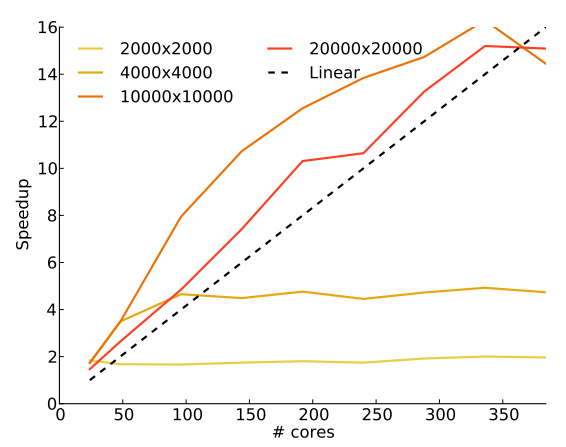

(d) Interpolate

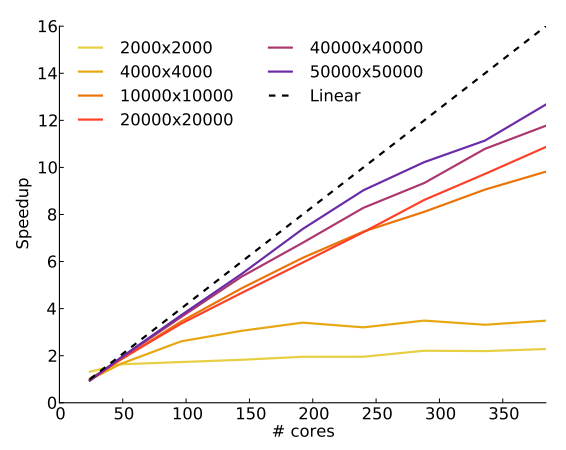

(g) Sobel

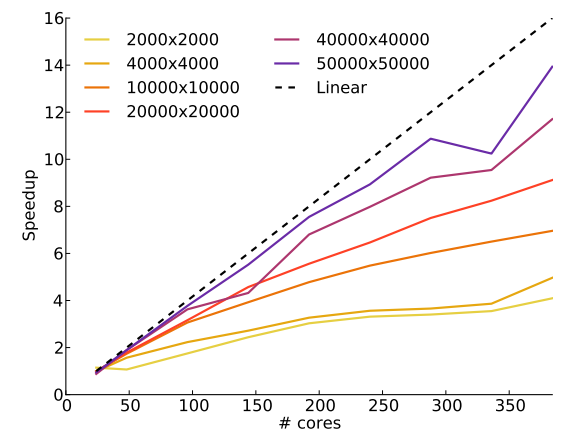

(b) Blur

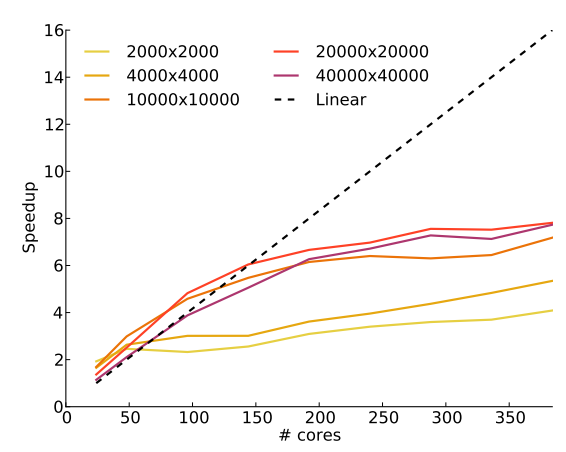

(e) Local Laplacian

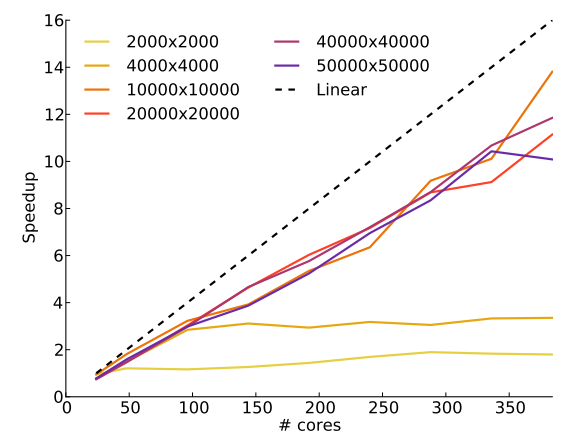

(h) Transpose

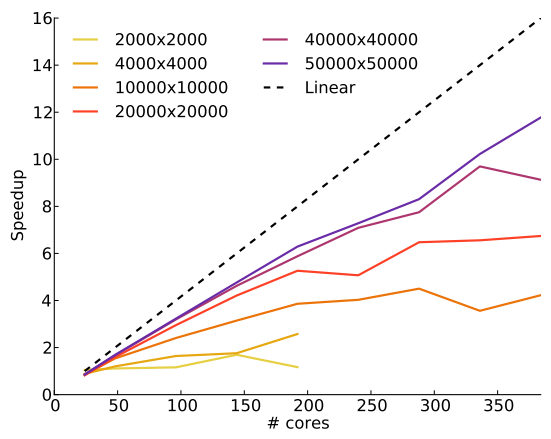

(c) Camera pipe

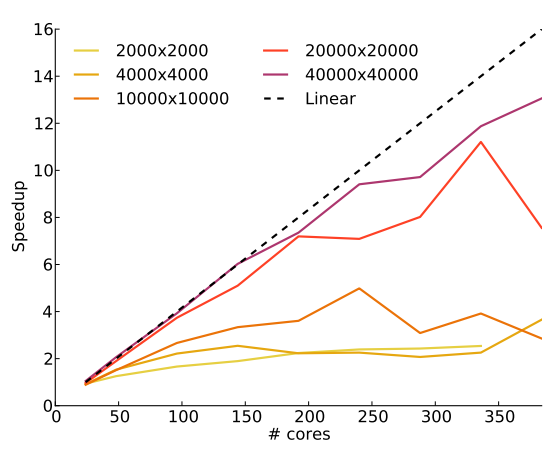

(f) Resize

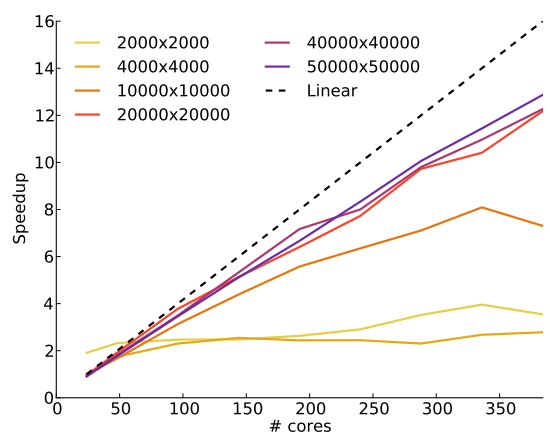

(i) Wavelet

Figure 5: Scaling results across all benchmarks with varying input sizes.

remote $\mathrm{L} 2$ cache, but these amounted to less than $0.1 \%$ of the total misses, so are not reported here.

We gathered these performance counters under four NUMA configurations of the 2D blur. In all cases, the schedule we used evaluated rows of the second blur stage in parallel (i.e blur_y .parallel (y)). For the distributed case, we simply distributed along the rows as well, i.e. blur_y. parallel (y) .distribute (y).

The four configurations were:

- Halide: Regular multithreaded Halide executing on all 24 cores.

- NUMA Local: Regular Halide executing on 12 cores on socket 0 , and memory pinned to socket 0 .

- NUMA Remote: Regular Halide executing on 12 cores on socket 0 , but memory pinned to socket 1 .

- Distr. Halide: Distributed Halide executing on all 24 cores, but with one MPI rank pinned to each socket.
For the "local" and "remote" NUMA configurations, we used the numactl tool to specify which cores and sockets to use for execution and memory allocation. The "local" NUMA configuration therefore is invoked with numactl $-\mathrm{m} 0-\mathrm{C} 0-11,24-35$, specifying that memory should be allocated on socket 0 , but only cores 0-11 (and hyperthread logical cores 24-35) on socket 0 should be used for execution. The "remote" configuration used numactl -m 1 -C $0-11,24-35$.

The results of these four scenarios are summarized in Table 5. The results indicate that approximately $50 \%$ of the last-level cache misses during regular multithreaded Halide execution required a fetch from remote DRAM. By using distributed Halide to pin one rank to each socket (the "distributed Halide" configuration), we achieve near-optimal NUMA performance, where $99.5 \%$ of LLC misses were able to be satisfied from local DRAM. 


\begin{tabular}{rccc} 
Config & $\begin{array}{c}\text { Total \# } \\
\text { Misses }\end{array}$ & $\begin{array}{c}\text { \% Local } \\
\text { DRAM }\end{array}$ & $\begin{array}{c}\text { \% Remote } \\
\text { DRAM }\end{array}$ \\
\hline Halide & $3.85 \times 10^{9}$ & $51.5 \%$ & $48.5 \%$ \\
NUMA Local & $2.36 \times 10^{9}$ & $99.6 \%$ & $0.4 \%$ \\
NUMA Remote & $3.48 \times 10^{9}$ & $3.6 \%$ & $96.4 \%$ \\
Distr. Halide & $2.29 \times 10^{9}$ & $99.5 \%$ & $0.5 \%$
\end{tabular}

Table 5: LLC miss resolutions during 23,000 $\times 23,000$ blur under several NUMA configurations.

\begin{tabular}{rrrr} 
Benchmark & Halide (s) & Distr. Halide (s) & Speedup \\
\hline bilateral grid & 9.772 & 10.116 & $0.966 \times$ \\
blur & 0.657 & 0.585 & $1.122 \times$ \\
camera pipe & 4.081 & 4.889 & $0.834 \times$ \\
interpolate & 2.588 & 1.822 & $1.420 \times$ \\
local laplacian & 11.826 & 10.003 & $1.182 \times$ \\
resize & 3.712 & 3.076 & $1.206 \times$ \\
sobel & 1.104 & 1.172 & $0.941 \times$ \\
transpose & 0.641 & 0.610 & $1.050 \times$ \\
wavelet & 0.673 & 0.712 & $0.944 \times$
\end{tabular}

Table 6: Runtime and speedup on a single node and the same number of cores with NUMA-aware distribution over two ranks, using each benchmark's maximum sized input.

Another item of note in the Table 5 is the total number of LLC misses from regular to distributed Halide in this scenario. This is due to the partially static, partially dynamic scheduling that occurs with the distributed schedule. In effect, each rank is statically responsible for the top or bottom half of the rows of the input. Then, parallelization using the dynamic scheduling happens locally over each half. Restricting the domain of parallelism results in better cache utilization on each socket, meaning many of the accesses that missed LLC in regular Halide become hits in higher levels of cache with distributed Halide.

Table 6 summarizes the benefit of using a distributed pipeline to form NUMA-aware static partitions. For each benchmark, we report the runtime of the maximum input size of the regular Halide pipeline versus the distributed pipeline. The distributed pipeline was run on a single node with the same number of cores, but one rank was assigned to each of the two sockets. The numbers are the median runtimes of 50 iterations.

While taking advantage of NUMA could also be done in the parallel runtime, our approach allows the distributed scheduling to generalize to handle NUMA-aware static scheduling, while maintaining the dynamic load balancing already present. This fits within the general Halide philosophy of exposing choices like these as scheduling directives: in effect, the distribute() directive can also become a directive for controlling NUMA-aware partitioning of computation.

\subsection{Scalability on Cori}

In order to support next-generation large-scale image processing, it is necessary for distributed Halide to scale to much higher core counts, such as what would used for on supercomputer-scale problems. Our testbed machine configuration is not quite representative of typical supercomputer architectures, not least due to the fact that our test network topology is fully connected. To measure how well our results generalize to a real supercomputer, we repeated the scalability measurements on "Cori," the newest supercomputer available at NERSC [3].

Cori is a Cray XC40 supercomputer, with a theoretical peak performance of 1.92 Petaflops/second. It has 1,630 compute nodes totalling 52,160 cores. Each compute node has two sockets, each of which is Intel Xeon E5-2698 v3 @ 2.3GHz. The network infrastructure is Cray Aries, with the "Dragonfly" topology. Each node has $128 \mathrm{~GB}$ of main memory. More details can be found at [3]. We ran our scalability tests up to a total of 64 nodes, or 2,048 cores.

The findings are summarized in Figure 7. The scalability of the benchmarks is similar to those observed on our testing machine. On 64 nodes, the blur benchmark achieves a $57 \times$ speedup for a parallel efficiency of $89 \%$, similar to the $86 \%$ efficiency on the 16 node test machine. The benchmarks that exhibited a falloff in scaling on our testing machine (such as local Laplacian) unsurprisingly do not scale on Cori. In the case of interpolate and resize, benchmarks that exhibited decent scaling on our testing machine, the falloff in scalability is due to strong scaling. We were unable to measure a single-node baseline for larger input sizes on these two benchmarks due to memory constraints. Thus, the curves quickly fall off as the limits of useful distribution are reached.

The transpose benchmark appears to display an accelerating scaling curve on smaller inputs, but these results should be taked with a grain of salt. We included small input sizes up to $20,000 \times 20,000$ for consistency across benchmark results, but the absolute difference in execution time between 32 and 64 nodes (1,024 and 2,048 cores) is less than 0.01 seconds, and the baseline is on the order of 0.1 seconds, as reported in Table 6 . Thus, the overall effect on execution time is nearly negligible from 32 to 64 nodes.

In general, a larger input size is required to see similar scaling at the 384 core count of our test machine. Most likely this is due to increased network contention during execution. In particular, the compute nodes assigned by the Cori job scheduler are not chosen based on locality, meaning the number of hops required for point-to-point communication can be much larger than on our test machine (which was fully connected). Additionally, Cori is a shared resource, meaning network contention with unrelated jobs could also have a non-negligible impact on scalability of these applications.

\section{Related Work}

Distributed Scheduling Finding an optimal allocation of tasks to distributed workers in order to minimize communication is an NPhard problem in general [14]. As such, there is a wealth of research devoted to approximate algorithms for finding task allocations to minimize communication, e.g. $[8,14,16,19,20,33]$. The distributed Halide compiler does not attempt to automatically determine distributed schedules. This follows the Halide philosophy in allowing the programmer to quickly try many different distributed schedules and empirically arrive at a high-performing distributed schedule. To semi-automate the search process, one can apply an autotuning approach as in [9].

In [15], the authors formulate a static polyhedral analysis algorithm to generate efficient communication code for distributed affine loop nests. This work uses a notion of "flow-out intersection flow-in" sets, derived using polyhedral analysis, to minimize unnecessary communication present in previous schemes. Our approach of required region intersection is similar to their approach. However, because our code generation can take advantage of domain-specific information available in Halide programs (for example, stencil footprints), our system has additional information that allows our code generation to be much simpler. A more general approach like flow-out intersection flow-in could be used, but would add unnecessary complexity.

Distributed Languages and Libraries In [23], an edge-detection benchmark was distributed on a network of workstations. The data partitioning scheme they adopted was to initially distribute all input required by each workstation, meaning no communication was re- 


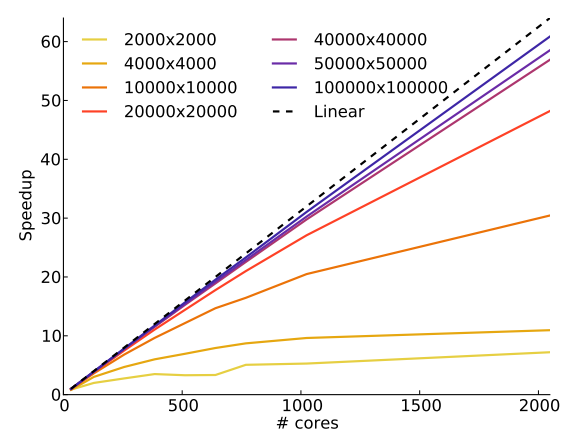

(a) Bilateral grid

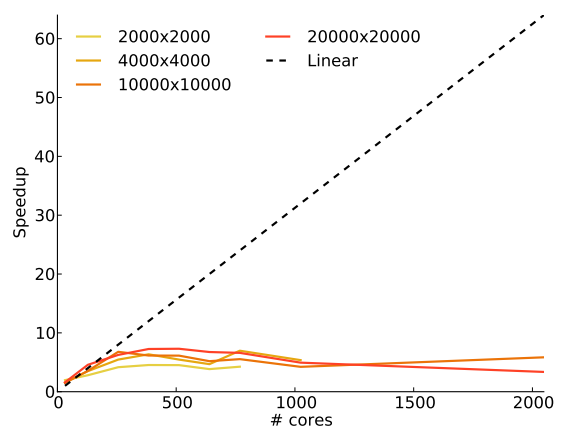

(d) Interpolate

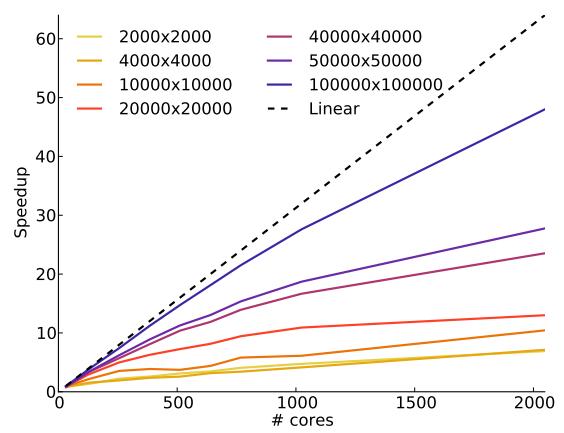

(g) Sobel

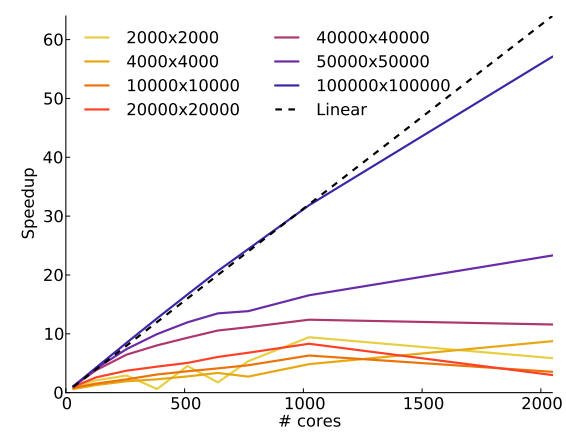

(b) Blur

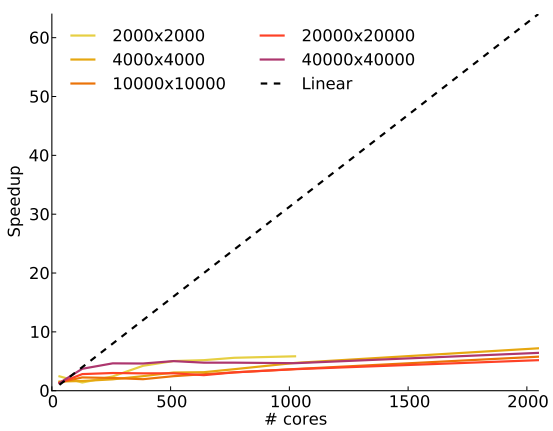

(e) Local Laplacian

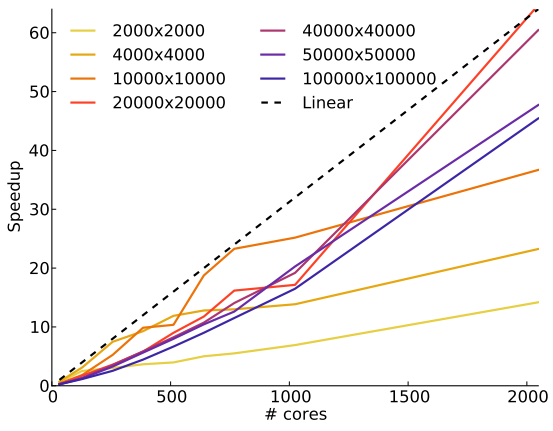

(h) Transpose

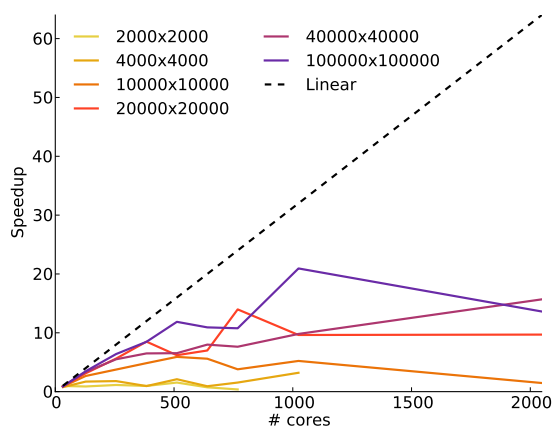

(c) Camera pipe

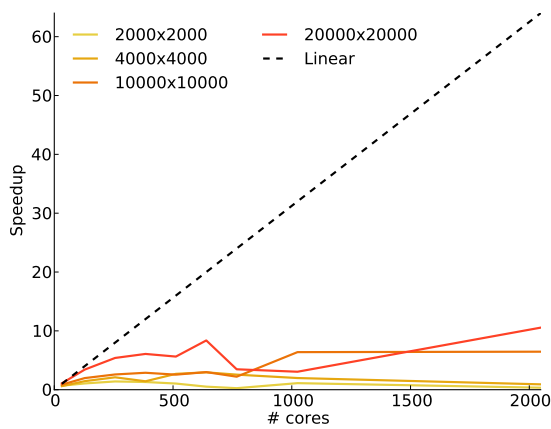

(f) Resize

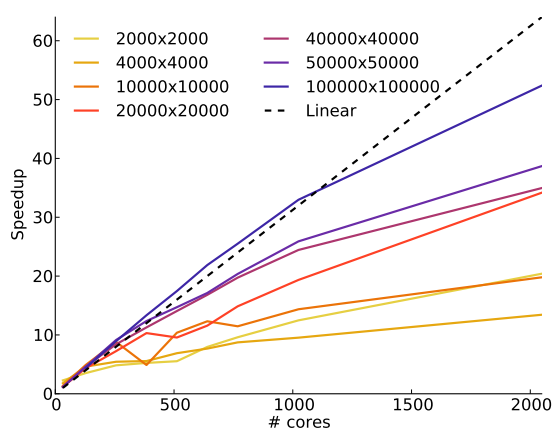

(i) Wavelet

Figure 7: Scaling results across all benchmarks with varying input sizes on the Cori supercomputer.

quired during execution. However, the software architecture in this work requires the distribution strategy to be implemented on their master workstation, and reimplementing a new data distribution in this architecture requires a non-trivial amount of work.

Some distributed languages such as X10 [11] and Titanium [18] include rich array libraries that allow users to construct distributed multidimensional structured grids, while providing language constructs that make it easy to communicate ghost zones between neighbors. However, exploring schedules for on-node computation requires rewriting large portions of the code.

DataCutter [10] provides a library approach for automatically communicating data requested by range queries on worker processors. Their approach requires generating an explicit global indexing structure to satisfy the range queries, whereas our approach maps data ranges to owners with simple arithmetic.
Image Processing DSLs Besides Halide, other efforts at building domain-specific languages for image processing include Forma [30], a DSL by Nvidia for image processing on the GPU and CPU; Darkroom, which compiles image processing pipelines into hardware; and Polymage [25], which implements the subset of Halide for expressing algorithms and uses a model-driven compiler to find a good schedule automatically. None of these implement distributedmemory code generation.

Stencil DSLs Physis [24] takes a compiler approach for generating distributed stencil codes on heterogeneous clusters. They implement a high-level language for expressing stencil code algorithms, and their compiler automatically performs optimizations such as overlap of computation and communication. Physis does not have analogy to Halide's scheduling language, meaning performance of a distributed stencil code completely depends on the au- 
tomatic compiler optimizations. Other stencil DSLs [13, 17, 21, 31] do not support distributed code generation, though they do generate shared-memory parallel code.

\section{Conclusions and Future Work}

Distributed Halide is a system that allows the programmer to quickly experiment to optimize a distributed image processing program both globally, in terms of communication of shared data, and locally in terms of on-node computation using a single unified language.

On nine image processing benchmarks, we demonstrated up to a superlinear $18 \times$ speedup on 384 cores distributed across 16 compute nodes and up to $57 \times$ speedup on 2,048 cores across 64 compute nodes on the NERSC Cori supercomputer. We also demonstrated up to a $1.4 \times$ speedup on single-node execution by mitigating NUMA architectural effects.

Looking forward, we plan to extend this work to support distribution across heterogeneous architectures with both CPUs and GPUs. As Halide already contains a backend capable of generating GPU code, this is a natural extension.

\section{Acknowledgments}

Thank you to Vladimir Kiriansky for help in measuring performance counters to understand the NUMA effects, and to Fredrik Kjølstad for numerous helpful discussions and reading early drafts of this paper. This work is supported in part by the DOE awards DE-SC0005288 and DE-SC0008923, and NSF XPS-1533753.

\section{References}

[1] Digitized Sky Survey. URL http://archive.stsci.edu/dss/.

[2] Canon 250 Megapixel Image Sensor, Press Release. URL http://www. canon.com/news/2015/sep07e.html.

[3] Cori Supercomputer System. URL http://www.nersc.gov/users/computational-systems/cori/.

[4] The OpenCV Library. URL http://code.opencv.org.

[5] Intel PMU Profiling Tools. URL https://github.com/andikleen/pmu-tools.

[6] E. H. Adelson, C. H. Anderson, J. R. Bergen, P. J. Burt, and J. M. Ogden. Pyramid methods in image processing. RCA engineer, 29(6): 33-41, 1984.

[7] A. Alexandrov, M. F. Ionescu, K. E. Schauser, and C. Scheiman. Loggp: incorporating long messages into the logp modelone step closer towards a realistic model for parallel computation. In Proc. of Parallel algorithms and architectures, pages 95-105. ACM, 1995.

[8] A. Amoura, E. Bampis, C. Kenyon, and Y. Manoussakis. Scheduling independent multiprocessor tasks. Algorithmica, 32(2):247-261.

[9] J. Ansel, S. Kamil, K. Veeramachaneni, J. Ragan-Kelley, J. Bosboom, U.-M. O'Reilly, and S. Amarasinghe. Opentuner: An extensible framework for program autotuning. In Proc. of Parallel architectures and compilation, pages 303-316. ACM, 2014.

[10] M. D. Beynon, T. Kurc, U. Catalyurek, C. Chang, A. Sussman, and J. Saltz. Distributed processing of very large datasets with DataCutter. Parallel Comput., 27(11):1457-1478, 2001.

[11] P. Charles, C. Grothoff, V. Saraswat, C. Donawa, A. Kielstra, K. Ebcioglu, C. von Praun, and V. Sarkar. X10: An object-oriented approach to non-uniform cluster computing. In Proc. of Object-oriented Prog., Systems, Languages, and Applications, OOPSLA '05, pages 519-538. ACM, 2005.

[12] J. Chen, S. Paris, and F. Durand. Real-time edge-aware image processing with the bilateral grid. In ACM Transactions on Graphics (TOG), volume 26, page 103. ACM, 2007.

[13] M. Christen, O. Schenk, and H. Burkhart. Patus: A code generation and autotuning framework for parallel iterative stencil computations on modern microarchitectures. In IPDPS, pages 676-687, May 2011.
[14] S. Darbha and D. Agrawal. Optimal scheduling algorithm for distributed-memory machines. Parallel and Distributed Systems, IEEE Transactions on, 9(1):87-95, 1998.

[15] R. Dathathri, C. Reddy, T. Ramashekar, and U. Bondhugula. Generating efficient data movement code for heterogeneous architectures with distributed-memory. In PACT, pages 375-386. IEEE, 2013.

[16] P.-F. Dutot, G. Mounié, and D. Trystram. Scheduling parallel tasks: Approximation algorithms. Handbook of Scheduling: Algorithms, Models, and Performance Analysis, pages 26-1, 2004.

[17] T. Henretty, R. Veras, F. Franchetti, L.-N. Pouchet, J. Ramanujam, and P. Sadayappan. A stencil compiler for short-vector SIMD architectures. In Proc. of Intl. conf. on supercomputing, pages 13-24. ACM, 2013.

[18] P. N. Hilfinger, D. Bonachea, D. Gay, S. Graham, B. Liblit, G. Pike, and K. Yelick. Titanium language reference manual. Technical report, Berkeley, CA, USA, 2001.

[19] K. Jansen. Scheduling malleable parallel tasks: An asymptotic fully polynomial-time approximation scheme. In Lecture Notes in Computer Science, pages 562-574. Springer Berlin Heidelberg, 2002.

[20] K. Jansen and L. Porkolab. General multiprocessor task scheduling: Approximate solutions in linear time. In Lecture Notes in Computer Science, pages 110-121. Springer Berlin Heidelberg, 1999.

[21] S. Kamil, C. Chan, L. Oliker, J. Shalf, and S. Williams. An auto-tuning framework for parallel multicore stencil computations. In IPDPS, pages 1-12, 2010.

[22] F. B. Kjolstad and M. Snir. Ghost cell pattern. In Proc. of Parallel Programming Patterns, ParaPLoP '10, pages 4:1-4:9. ACM, 2010.

[23] X. Li, B. Veeravalli, and C. Ko. Distributed image processing on a network of workstations. Intl. Journal of Computers and Applications, 25(2):136-145, 2003.

[24] N. Maruyama, T. Nomura, K. Sato, and S. Matsuoka. Physis: an implicitly parallel programming model for stencil computations on large-scale GPU-accelerated supercomputers. In High Performance Computing, Networking, Storage and Analysis, pages 1-12. IEEE, 2011.

[25] R. T. Mullapudi, V. Vasista, and U. Bondhugula. Polymage: Automatic optimization for image processing pipelines. In Proc. of Architectural Support for Programming Languages and Operating Systems, ASPLOS ' 15, pages 429-443. ACM, 2015.

[26] D. K. Panda et al. OSU Microbenchmarks v5.1. URL http://mvapich.cse.ohio-state.edu/benchmarks/.

[27] S. Paris, S. W. Hasinoff, and J. Kautz. Local Laplacian filters: edgeaware image processing with a Laplacian pyramid. ACM Trans. Graph., 30(4):68, 2011.

[28] J. Ragan-Kelley. Decoupling Algorithms from the Organization of Computation for High Performance Image Processing. PhD Thesis, MIT, 2014.

[29] J. Ragan-Kelley, C. Barnes, A. Adams, S. Paris, F. Durand, and S. Amarasinghe. Halide: A language and compiler for optimizing parallelism, locality, and recomputation in image processing pipelines. In Proc. of Programming Language Design and Implementation, PLDI '13, pages 519-530. ACM, 2013.

[30] M. Ravishankar, J. Holewinski, and V. Grover. Forma: A DSL for image processing applications to target GPUs and multi-core CPUs. In Proc. of General Purpose Processing Using GPUs, GPGPU-8, pages 109-120. ACM, 2015.

[31] Y. Tang, R. A. Chowdhury, B. C. Kuszmaul, C.-K. Luk, and C. E. Leiserson. The Pochoir stencil compiler. In Proc. of Parallelism in Algorithms and Architectures, SPAA '11, pages 117-128. ACM, 2011.

[32] J. S. Vetter and M. O. McCracken. Statistical scalability analysis of communication operations in distributed applications. In Proc. of Principles and Practices of Parallel Programming, PPoPP '01, pages 123-132. ACM, 2001.

[33] S. Wholey. Automatic data mapping for distributed-memory parallel computers. In Proceedings of the 6th international conference on Supercomputing, pages 25-34. ACM, 1992. 